(C2005 IEEE. Personal use of this material is permitted. However, permission to reprint/republish this material for advertising or promotional purposes or for creating new collective works for resale or redistribution to servers or lists, or to reuse any copyrighted component of this work in other works must be obtained from the IEEE. 


\title{
Holonic Multi-Agent System Complemented by Human Disease Ontology Supporting Biomedical Community
}

\author{
M. Hadzic, E. Chang \\ Curtin University of Technology, School of Information Systems, GPO Box U1987, Perth 6845, Western Australia, Australia \\ e-mail: (maja.hadzic, elizabeth.chang)@cbs.curtin.edu.au
}

\begin{abstract}
The medical milieu is an open environment characterized by a variety of distributed, heterogeneous and autonomous information resources. Coordination, cooperation and exchange of information is important to the medical community. This paper presents an Ontology-based Holonic Multiagent System that combines the advantages of the holonic paradigm with multi-agent system technology and ontology design, in order to realize a highly reliable, adaptive, scalable, flexible and robust diagnostic system for diseases.

We design a new ontology, called Generic Human Disease Ontology (GHDO), for the representation of knowledge regarding human diseases. The concepts of the GHDO ontology are organized into the following four dimensions: Types, Symptoms, Causes and Treatments of human diseases. The holonic multi-agent system uses this common GHDO ontology for purpose of query formulation, information retrieval and information integration. This intelligent dynamic system provides opportunities to collect information from multiple information resources, to share data efficiently and to integrate and manage scientific results in a timely manner. We believe such a technique is expected to become the norm once existing resources (e.g. disease databases) will have become unlocked semantically through annotation with a shared ontology.
\end{abstract}

Index Terms-holonic multi-agent systems, ontology-based multi-agents systems, human disease ontology, intelligent information retrieval.

\section{INTRODUCTION}

Traditionally, the knowledge base in biomedicine has resided within the heads of experienced scientists and doctors, who have devoted much study to become experts in their particular domain of study. This approach worked well in the past when the flow of data was not so great as to overwhelm the expert. However, this situation is rapidly changing. New modern techniques are providing huge, rapidly accumulating amounts of information.

Good predictions can only be made against a knowledge base. The bigger the knowledge base the better the predictions that can be made. However, the size of the existing knowledge base is too large for any human to assimilate. Therefore, predictions are only being made against a small subset of the available knowledge. Unfortunately, in most cases some important information is being neglected. There is a need to create systems that can apply all available knowledge to biomedical data. Such systems could play a crucial role in filtering the flood of data to the point where human experts could again apply their knowledge sensibly.
In order to make all these data really useful, one needs tools that will access and retrieve exactly the information one needs. The available online information needs to be intelligently queried. We have focused on the application domain of human disease research and control. A characteristic of the domain is that trusted databases exist but their schemas are often poorly documented, if at all. The management of resources and services is important in the biomedical community and associated smaller communities of people committed to a common goal. The network of biomedical databases forms a loose federation of autonomous, distributed, heterogeneous data repositories ready for information integration.

Our research is centred on the task of formalizing and combining the knowledge regarding human diseases into a single coherent unifying framework. We aim to develop a methodology to access, extract and manipulate biomedical information from various information resources.

In this paper, we introduce an ontology-based holonic multi-agent model for the information retrieval of biomedical knowledge related to human diseases. The ontology is realized in holonic multi-agent system designed to aid medical researchers, physicians and patients in retrieving relevant information regarding human diseases. But we believe that the way we approach our problem is applicable to other knowledge domains as well.

This paper is structured as following. In Section 2, we discuss related work in the biomedical domain. In Section 3 , we describe basic assumptions taken in the process of system construction. In Section 4, we describe how we designed Generic Human Disease Ontology (GHDO). In Section 5, we describe GHDO-based Holonic Multi-agent System (GHMS) and different types of agents within the system. In Section 6, we describe query processing and information integration mechanism within the system. Finally, in Section 7, we conclude and provide our final remarks.

\section{RELATED WORK}

Guardian is a knowledge-based system for monitoring and diagnosis of post-cardiac surgery patients in an intensive-care unit. It is designed as an autonomous agent with a flexible architecture and adaptive mode of operation in which several different algorithms cooperate to reach diagnoses under hard realtime conditions [1].

Agent Cities [2] is a multi-agent system (MAS) designed and constructed which is composed of agents that provide 
medical services. The MAS contains agents that allow the user to search for medical centers satisfying a given set of requirements, to access his/her medical record or to make a booking to be visited by a particular kind of doctor. The main aim of this project is the construction of a worldwide, publicly accessible network of agent platforms. Each agent platform will support agents that offer services similar to those that can be found in a real city (facilities, amenities, information and commercial services).

AADCare agent architecture [3] comprises multiple layers of knowledge, a working memory, a communications manager and a human-computer interface. The agents themselves are implemented using the layered architecture which combines a number of $\mathrm{AI}$ and agent techniques. The three layers of knowledge which form the key part of the AADCare architecture are domain knowledge (a knowledge base covering specific medical domains), inference knowledge (generic, declarative inference rules which specify inference relations between domain knowledge, existing patient information and possible new data) and control knowledge (applies the inference knowledge to the domain knowledge in order to generate new inferences whenever new data is added to the working memory).

BioAgent [4] is a mobile agent system suitable to support bioscientists during the process of genome analysis and annotation. An experiment is seen as a coordinated execution of multiple tasks or activities, where one task consists in visiting a set of data repositories distributed over multiple locations and for each location extracting certain information, if any. An agent is associated to the given task and it travels among multiple locations, called places, and at each location performs its mission. At the end of the trip, an information integration procedure takes place before the answer is deployed to the user.

Holonic Medical Diagnostic System (HMDS) is a medical diagnostic system [5]. This system combines the advantages of the holonic paradigm, multi-agent system technology and swarm intelligence in order to realize Internetbased diagnostic system for diseases. Each agent has a certain responsibility. Some agents may represent experts on a broader field of diseases while others may be experts on (occurrences of) one specific disease. Like ants in an ant colony, the proposed agents collaborate in order to provide a reliable medical diagnosis.

Guardian is designed as an autonomous agent while Agent Cities and AADCare are designed as multi-agent architecture. BioAgent and HMDS are systems that make use of mobile agents. The use of agents within the medical community is quite encouraging. Yet, none of the above mentioned agent architectures make use of the ontologies. As a rich form of domain knowledge representation, ontologies were in the first place brought into the computer and information society for the purpose of being used by agent systems. We propose an ontology-based holonic multi-agent system in which ontologies are used for the purpose of intelligent information retrieval.

\section{ASSUMPTIONS}

We assume that each disease is uniquely described by its different types, symptoms, causes and treatments, as shown in Figure 1. By specifying all of the four 'dimensions', it is possible to characterize a specific disease.

It is impossible for two different diseases to have same characteristics describing their disease types, symptoms, causes and treatments. At least one of the mentioned four components needs to be different in case of different diseases.

Let $\mathrm{D}, \mathrm{S}, \mathrm{C}$ and $\mathrm{T}$ describe disease types, symptoms, causes and treatments of a diseases respectively. For the two diseases Disease $\mathrm{k}$ and Disease 1, we have that:

If Disease $\mathrm{k}=$ Disease 1 , then

$(\mathrm{D} \mathrm{k}=\mathrm{D} 1) \wedge(\mathrm{S} \mathrm{k}=\mathrm{S} 1) \wedge(\mathrm{Ck}=\mathrm{C} 1) \wedge(\mathrm{T} \mathrm{k}=\mathrm{T} 1)$

If Disease $\mathrm{k} \neq$ Disease 1 , then

$(\mathrm{D} \mathrm{k}=\mathrm{D} 1) \vee(\mathrm{Sk}=\mathrm{S} 1) \vee(\mathrm{Ck}=\mathrm{C} 1) \vee(\mathrm{T} \mathrm{k}=\mathrm{T} 1)$

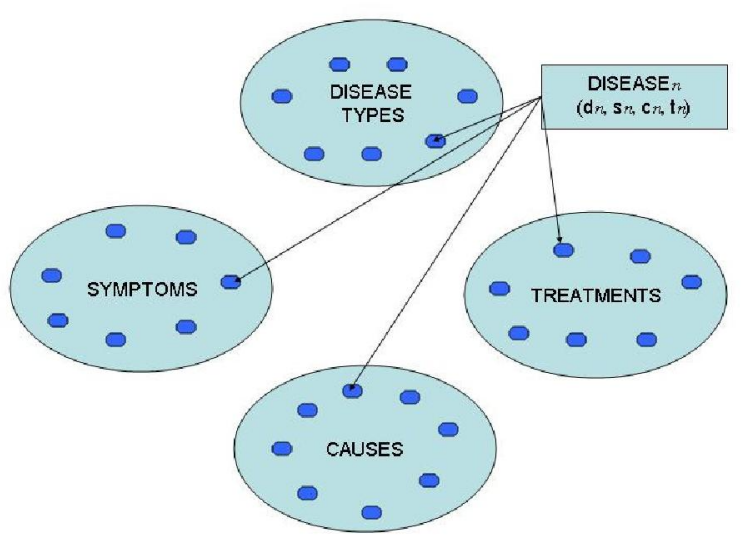

Fig. 1. A disease is uniquely specified by the four components

On the basis of this assumption, we develop an ontology to cover the information according to the four 'dimensions'.

\section{GENERIC HUMAN DISEASE ONTOLOGY (GHDO)}

Different terminologies and ontologies have been developed in many different areas of medical domain. In order to obtain some uniformity across different ontologies, definitions from other published and consensual ontologies can be reused [6]. Lots of applications already use the existing terminologies like UMLS [7] and LinkBase [8]. Rather then creating a new terminology, we decided to use concepts from existing ontologies. The way that these concepts are organized within the existing ontologies is not suitable to be used by our system. The four different 'dimensions' (subontologies) of Generic Human Disease Ontology (GHDO) can serve as a reference points against which the concepts from the existing medical ontologies can be reorganized, aligned and merged. So, we use terminology from existing 
ontologies but organize these concepts in a way that can be used by our system.

We identified two main user categories of the system:

- medical researches that are mainly interested in causes of a disease, and

- $\quad$ physicians and patients that are faced with situation of a disease and are mainly interested in symptoms and treatments of a disease.

Having this two users categories in mind and on the basis of assumption described in the previous section, we construct Generic Human Disease Ontology (GHDO) [9]. The GHDO has four main branches:

- disease types, describing different types of a disease;

- phenotype, describing symptoms of a disease;

- causes responsible for that disease which can be genetic and/or environmental;

- treatments, giving an overview of all treatments possible for a particular disease;

Top-level hierarchy of GHDO is illustrated in Figure 2.

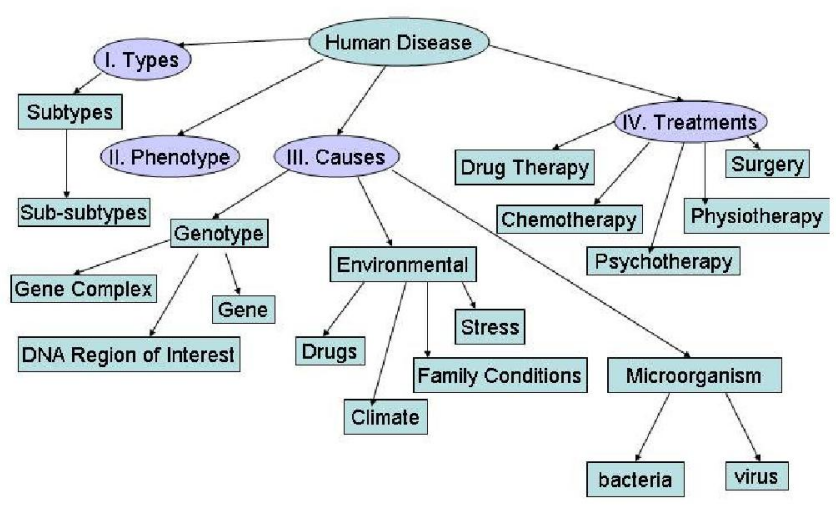

Fig. 2. Top-level hierarchy of GHDO

The information presented in this figure state that a disease may have different types that also further may be divided into subtypes and sub-subtypes. For each disease, there is a corresponding phenotype or observable characteristics of an ill individual, namely symptoms of a disease. Each disease is caused by cause(s) which can be genetic (genotype), environmental or a disease may be caused by a microorganism. Genetic causes can be a mutated gene, a complex of genes or DNA region of interest. DNA region of interest is a region in the DNA sequence that potentially contains a gene responsible for the disease. This region needs to be further examined in order to correctly locate the mutated gene. Environmental causes of a disease can be stress, climate, drugs or family conditions. Microorganisms that may cause a disease may be virus or bacteria. Possible treatments for a disease can be drug therapy, chemotherapy, surgery, psychotherapy or physiotherapy.

A user may only be interested in a part of information presented by GHDO. Accordingly to user's request, the overall problem to be solved by the multi-agent system is constructed from GHDO [10]. This step results in Specific Human Disease Ontology template, SHDO template. For example, a user is interested in symptoms (phenotype) and causes of a specific disease. The query is then constructed as SHDO template that is composed of two sub-ontologies: Symptoms and Causes sub-ontologies. The significant information is retrieved by different agents of the system and added upon this SHDO template. This step results in Specific Human Disease Ontology, SHDO. SHDO is presented to the user as answer to his/her query.

\section{GHDO-BASED HOLONIC MULTI-AGNET SYSTEM (GHMS)}

In this section, we illustrate how we use GHDO to support information retrieval process within holonic multiagent structure. The GHDO-based Holonic Multi-Agent Structure (GHMS) (shown in Figure 3) is a nested hierarchy of four holarchies (hierarchies of different agents). Each of the four GHDO dimensions (Disease types, Symptroms, Causes and Treatments) is associated with one holarchy. Information is interpreted and analyzed at higher level, while collection of data happens at lower level of holarchies.

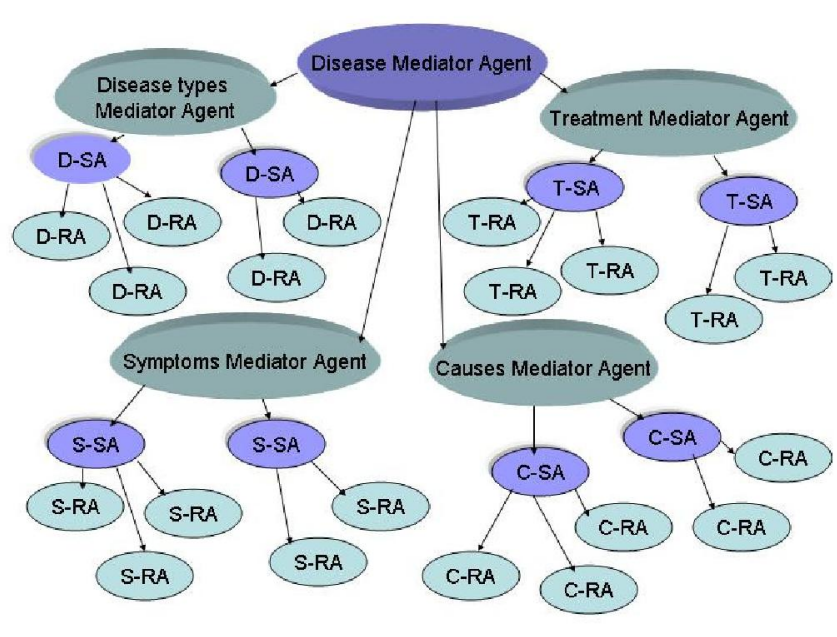

Fig, 3. GHMS structure

Disease Mediator Agent (DMA). Each holarchy has a single entry point, named Mediator Agent. GHMS has as main entry point, Disease Mediator Agent (DMA). On the basis of SHDO template, DMA decides which of the four holarchies needs to be deployed.

Mediator Agents (MAs). Each branch of the main entry point of GHMS (DMA) has its own mediator agents, respectively Disease Types, Symptoms, Causes and Treatments Mediator Agents (D-MA, S-MA, C-MA and T-MA). Their task is to decide what other subordinate Specialist Agents (DAs) or Representative Agents (RAs) need to be activated in order to retrieve information requested by the user.

Specialists Agents (SAs). Holarchy inner nodes represent Specialist Agents (SAs). They represent decision mak- 
ers and are specialists on a specific topic of corresponding sub-ontology. We differentiate Disease types, Symptoms, Causes and Treatments Specialists Agents (D-SA, S-SA, CSA and T-SA). Also, after subordinate agents (RAs) have returned their data, SAs interpret, compare, and evaluate this data in order to define a proper ranking among them. Not only defining a proper ranking among all the delivered data, but an important function of a SA is also to interpret incoming data and come to a conclusion on whether there is sufficient information retrieved in regard to SHDO template. If not, SA has to decide - on the basis of the delivered information - whether it makes sense to consult other RAs.

Representative Agents (RAs). The leaves are so-called Representative Agents (RAs). We differentiate Disease types, Symptoms, Causes and Treatments Representative Agents (D-RA, S-RA, C-RA and T-RA). Each RA is an expert on a lowest level concept within the ontology. Note that RAs differ from SAs in that they need to recognize the significant information inside appropriate database and retrieve that information. This information is then passed over to SAs which will do analysis, comparison and assembly of the retrieved information. Selected information is then passed over to corresponding mediator agents.

\section{QUERY PROCESSING AND INFORMATION INTEGRATION WITHIN THE SYSTEM}

Query in the form of SHDO template is send to Disease Mediator Agent, DMA. DMA partition SHDO template into four sub-ontologies templates: AOe (disease types), AOe (symptoms), AOe (causes) and AOe (treatments). The resulting sub-templates have form of AOe (sub-ontology) and are sent to corresponding Mediator Agents, MAs. (The notation AOe stands for agent's external ontology component. There is also AOi that represents agent's internal ontology component). MA receive also information regarding other sub-ontologies templates, but it only needs to process its own. For example, Causes-Mediator Agent (C-MA) receives the four sub-ontology templates but it only further processes AOe (causes) template.

The AOe (sub-ontology) is further partitioned by MAs into sub-sub-ontologies and parts of them, such as AOe (drug therapy), AOe (surgery), AOe (chemotherapy), AOe (genetic), AOe (environmental), etc. The resulting sub-subtemplates are of the form AOe (sub-sub-ontology). The process is shown in Figure 4. According to the templates, tasks are assigned to Specialist Agents and Representative Agents. SAs and RAs are also aware of tasks assigned to other holarchy agents as they received information regarding other parts of SHDO template. In process of assembling retrieved information, MAs combine information coming from RAs and SAs in the form AOe (sub-sub-ontology), and present it to DMA as a single unit of the form AOe (subontology).

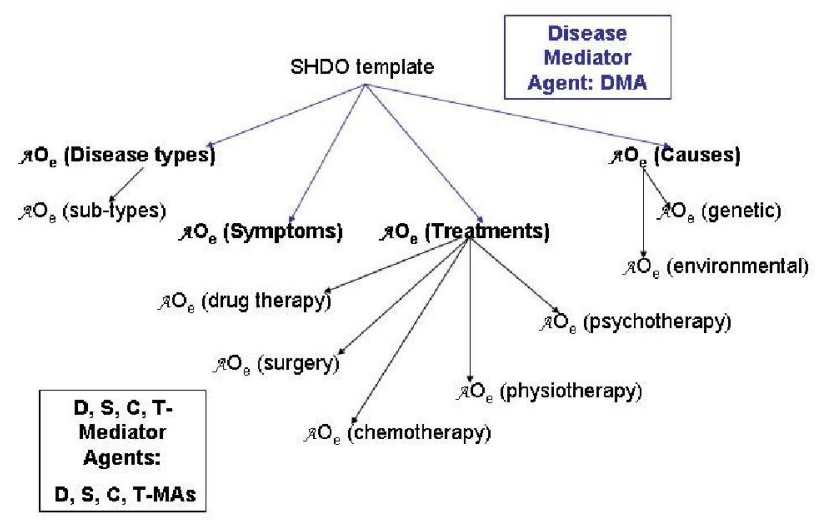

Fig. 4. Partitioning of SHDO template

During information integration process, Disease Mediator Agent performs two different types of matching. First one is matching of the originally defined SHDO template with the incoming information. Four different AOe of four different sub-ontologies are combined together by DMA resulting in Specific Human Disease Ontology (SHDO): AOe (disease types) $\cup$ AOe (symptoms) $\cup$ AOe (causes) $\cup$ AOe (treatments) $=$ SHDO. DMA relies not only on its knowledge but also on experiences it made in the past. For this reason, latest version of SHDO regarding same disease requested by some other user before, are saved in a pattern store, making it possible to do second type of matching. If a difference is found, new SHDO should be checked for its consistency. If difference is consistent, latest version of SHDO should be saved in a pattern store and used next time for matching. It may be possible that relevant data/examinations are missing and that more information may be needed. In this case, more agents need to be activated until the process is completed.

\section{CONCLUSION AND FUTURE WORK}

In this paper, we present ontology-based holonic multiagent system that unifies the advantages of multi-agent system technology with those of an integrated ontology for the purpose of representing the active knowledge about human disorders. The system is designed to aid medical researchers, physicians and patients in retrieving relevant information regarding human diseases. But we believe that the way we approach our problem is applicable to other knowledge domains as well.

The four ontology 'dimensions' (disease type, phenotype, causes and treatments) are each built for a different purpose and are orthogonal to each other. The 'Disease Types' sub-ontology is more a classifying ontology and is strongly hierarchically supported. It does not provide a user with much scientific information. This ontology is based on classification. The 'Phenotype' sub-ontology is more descriptive than the others and is based on observation and diagnosing characteristics of ill individuals. The 'Cause' sub-ontology is providing a user with scientifically proven facts and is strongly based on scientific research. The 'Treatment' sub-ontology is a combination of classifying and research ontology. Designing new drugs is research work but, for example all the discovered drugs can be hierarchically classified. All four 'dimensions' are different 
from each other and each 'dimension' is unique. But jointly they give an overall picture and a good overview of knowledge about a human disease.

Ontologies represent domain knowledge and are used for the purpose of intelligent information retrieval. The multiagent system provides system dynamic, distributed collaborative platform and easy access to resources. The information agents are specialized in retrieving information from different information resources, analyzing the obtained data, filtering redundant or irrelevant information, selecting the relevant information and presenting it to the user.

The innovation in our work lies in combination of holonic architectures, multi-agent technology for managing unstructured biomedical information into structured disease information for end users and use of ontologies which act as spinal cord of the system. Involving agents to integrate dispersed knowledge sources into the ontology template results in a powerful mechanism for dynamic building of new knowledge. However, lots of work still remains, such as implementation of local agent interactions, security concerns and development of user view interfaces.

\section{REFERENCES}

[1] J. E. Larsson, B. Hayes-Roth, D. Gaba, 'Guardian: Final Evaluation', Knowledge Systems Laboratory Report No. KSL-96-25, 1996.
[2] A. Moreno, D. Isern, "A first step towards providing health-care agent-based services to mobile users", in Proceedings of the first international joint conference on autonomous agents and multiagent systems (AAMAS'02), 2002, pp. 589-590.

[3] J. Huang, N. R. Jennings, J. Fox, "An Agent-based Approach to Health Care Management", International Joumal of Applied Artificial Intelligence, vol. 9, no. 4, 1995, pp. 401-420.

[4] E. Merelli, R. Culmone, L. Mariani, "BioAgent: A Mobile Agent System for Bioscientists", in Proceedings of the Network Tools and Applications in Biology Workshop Agents in Bioinformatics (NETTABO2), 2002.

[5] M. Ulieru, "Internet-Enabled Soft Computing Holarchies for eHealth Applications", New Directions in Enhancing the Power of the Internet, 2003, pp. 131-166.

[6] N.F. Noy, M.A. Musen, "PROMPT: Algorithm and Tool for Automated Ontology Merging and Alignment", in Proceedings of the seventeenth national conference on Artificial Intelligence (AAAI2000), 2000, pp. 450-455.

[7] O. Bodenreider, "The Unified Medical language System (UMLS): integrating biomedical terminology", Nucleic Acids Res, vol. 32, no. 1, 2004, pp. 267-270.

[8] F. Montyne, "The importance of formal ontologies: a case study in occupational health", in Proceedings of the international workshop on Open Enterprise Solutions: Systems, Experiences, and Organizations (OES-SEO2001), 2001.

[9] M. Hadzic, E. Chang, "Ontology-based Support for Human Disease Study", in Proceedings of the 38th Hawaii International Conference on System Sciences (HICSS-38), 2005.

[10] M. Hadzic, M. Ulieru, E. Chang, "Ontology-Based Holonic Diagnostic System for the Research and Control of New and Unknown Diseases", in Proceedings of the IASTED International Conference on Biomedical Engineering, 2005. 